\title{
Cheap Deployable Networked Sensors for Environmental Use
}

\author{
Alex Antunes \\ Capitol Technology University
}

Summary: We profile the utility and limits in deployment of inexpensive disposable sensor networks by amateurs to achieve environmental monitoring goals. Four current technology movements - PC-ona-chip processors such as Arduino, prebuilt sensors, 3-D printing, and Wi-Fi and mesh networking enable rapid sensor platform creation and make it easy for non-specialists to create general sensorbearing platforms. Deployment of a cluster of multiple sensor-bearing platforms is, however, communications-limited in terms of both range and number of devices supported, and generally requires a base station with internet access. We examine how inexpensive technology supports scenarios for short-term environmental modelling by average citizens.

\section{Introduction}

When an environmental crisis or disaster occurs, there is a need is to deploy a large number of sensors quickly over a large area to provide data for a short period of time to a local controller. Scenarios include mapping the spread of a wildfire, tracking the rise of a flood, and one-time data measurement of a potential contamination source. While most crises currently rely on state-supported agencies (first responders and authorised scientific agencies) to provide data to the public, we assert that the technology is already in place to enable appropriate crisis, ad hoc, and steady state data collection at the local, private, and amateur level. The primary limitation is not hardware or skill, but effective implementation of communications to minimise the cost-per-sensor and ensure effective data collection from a dispersed set of sensors.

Key for these sensors is the engineering limits requiring low cost, minimal technical skills, and ability to rapidly deploy with or without permission from state agencies. Internet access combined with smart devices can enable use of telecomm networks to create ad hoc sensor data collection networks over the channels currently used for voice, email and web browsing. By using conventional internet, this allows creation of smart sensor networks at either an official or activist level.

Smart devices and 'the internet of things' suggest ordinary appliances be made net-accessible, 3-D printing enables rapid construction of simple prototypes, and Arduino- and Pi-based kits let teams build inexpensive sensor rigs. The combination of these technologies enables creation of customised inexpensive one-use sensors that can be deployed or scattered over a target area. Like buoys on the ocean, data can be sampled in-situ at many places to provide a 
detailed geographic picture of wildfire advances, pollution or toxin levels over a disputed site, rainfall in flood country, and other simple measurables.

The downside is the 'landmine' problem; tracking and disposing of your sensors to avoid adding to environmental woes. We discuss how proliferation of easy-to-deploy 'land buoys' can advance scientific and activist goals by combining inexpensive off-the-shelf technology with internet access to advance environmental causes and disaster assessment. However, citizen monitoring must also be aware of social concerns in the pursuit of data.

This paper lists situation drivers for short-term environmental data collection, followed by the operational limits. Several existing sensor deployments are presented, followed by possible future scenarios. The paper lists a prototype generic build, then discusses communications issues and communications topologies. After survivability and recover concerns are discussed, the paper concludes with a review of the frequency limitations and potential abuse likely in pursuit of these data gathering goals.

\section{Situation drivers}

The conventional image of a scientific sensor package, remote sensing robot, or surveillance drone assumes two-way communication that allows the device to be modified during use by a skilled remote operator. Such 'smart' sensors can be manipulated and reconfigured in real time to optimise the data return. Deployable pre-configured non-adjustable 'dumb' sensors lacking two-way communication and with no movement or positioning capability - are not yet a commercial segment and require custom building. Such sensors do not require advanced degrees or large funding to create. By analogy, a weather satellite can obtain temperature data for a hemisphere of the entire Earth from space over broad areas of the sea, but we require inexpensive temperature sensors on cheap buoys to provide more detailed mile-by-mile measurements of ocean temperature variance. For situations requiring sampling of a small spatial area with many data points, deployment of multiple ground-based sensors is required.

A low time sampling rate and simple low-resolution detector images are acceptable in favour of being able to sample a local geographic region with high spatial sampling, especially for transient events or items not available by satellite due to being in economically minor areas. On-site small-scale data collection is a sub-range in terms of area (small) and resolution (high) combined with high time resolution (24 hours, 7 days at week at minutes or hours sampling).

This produces a level of detail that satellites can't see from Geosynchronous orbit (GEO) using Earth-observing missions, which have spatial resolution no smaller than $1 \mathrm{~km}$ in area. Monitoring often requires data taken more frequently than with satellites such as the Afternoon Constellation of international earth-observing satellites (dubbed the 'A-train') that pass overhead over 90 minutes.

For in-situ sensors, conventional military, commercial and scientific work tends towards robust multi-capability sensor platforms with extensive software support, dedicated 
communications, and a correspondingly high price. However, disposable inexpensive sensors exist and are proliferating. The primary bottleneck is access to communications from the environmental site to the data collection centre. We look at cases that need sensors, evaluate cost and design issues for sensor design and deployment, calculate coverage areas, and discuss deployment and communications issues that, if resolved, leads to good science.

Communications becomes the limiting factor. Middleton (2014) discusses the geographic limits in availability of mobile broadband and wireless access in Australia, noting "there are no comprehensive statistics that track WiFi users" and that "service is available on a patchwork basis, with many different providers offering WiFi coverage in specific geographic locations, sometimes for free, sometimes not". Middleton suggests future user access can be geographically broadened, in part, by allowing access over both licensed (commercial) and licence-exempt (non-commercial) spectrum.

This follows the model of the 'Internet of Things' (IoT), a scenario where ordinary devices such as toasters are Internet-enabled to allow data collection and remote operations. The limitation is no longer cheap hardware availability or short-range data transmission, but the need to network a hub or base controller to get the collected sensor net data from the work site via the larger internet to the lab.

\section{Potential scenarios}

Potential implementation of citizen- and amateur-deployed sensors requires an assessment of cost per sensor, expected coverage area desired, and communications and network support required to deliver the data to the user. To maintain the low cost per sensor, we continue with the model of using non-mobile wireless sensors that transmit repeated data points for the desired measureable, require no real time commanding, and only gather data for a brief time (hours to days).

Tasks can include monitoring of natural disaster events such as fires, volcanos, ice, and flood. Industrial monitoring includes tracking industrial and chemical activity, noise levels, or radiation levels. Farm and environmental assessment of small regions are a third category. Purely scientific studies of dry and wet regions are also a category favouring small inexpensive deployable sensors. Urban situations also can be monitored. In all cases, the data is transmitted away from the target site to a remote user, necessitating a long-distance communication pathway via internet, amateur radio, or GSM.

Sample crises include fire rehabilitation, fuel maps to predict fire spread, volcano eruption assessment, flood assessment, and ice breakup change detection. Chemical pollution tracking includes on land or in bodies of water, either to detect whether contamination exists or to monitor changes in concentrations. Agricultural uses include monitoring rain and water levels, and is particularly documented in the previous examples, as this is a commercially viable area. Marine biology uses in littoral zones, watersheds, and wetlands, as well as arid environments including desert, mountain and ice climes. In addition to the previous examples 
of urban heating, sensors can be deployed to track noise levels, building vibration due to traffic, light levels for trees in city environments, $\mathrm{CO} 2$ or pollutant mapping, and even utilisation rates of roads or parks as determined by noise levels.

The operational limits for these scenarios require that

(a) the sensor data is gathered from a distance of at least $1 \mathrm{~km}$ from the sensor deployment and

(b) that multiple sensors are able to return a position via GPS data, a timestamp, and the sensor value gathered.

Communication via internet requires network access, as does GSM use. Communications via radio use must follow national codes, such as the Federal Communications Commission (FCC) in the US and the Australian Communications and Media Authority (ACMA) in Australia.

Licence compliance is one of the communications bottlenecks. Because spectrum is regulated, citizens are not allowed to arbitrarily boost the signal from low-power off-the-shelf devices, install repeaters across public land, or access the radio spectrum except in narrowly permitted bands such as the channels allocated to amateur ham radio, that have their own licensing requirements for use.

Off-the-shelf radio transmitters include the XBee and other short-range devices, conventional Wi-Fi devices intended to be received by a Wi-Fi hub, and licensed radio transmitters at multiple frequencies. Our communications model is simplified in that we require sensor nodes to transmit only, and do not require real time commands be transmitted to the sensors. Thus we require only sensor transmitters with no receivers, and likewise any base hub need only receive, not transmit. Devices with inherent mesh capability, where each device may reroute signals from adjacent nodes as well as its own data, do require transceiver capability but are allowable in our scenarios if their price point and ease of development comply with our requirements.

\section{Existing hardware}

The use of drones and robots for measurement is one potential solution, which is worth noting in the context of these scenarios. The marketing of items for the 'maker' and Do-It-Yourself (DIY) hobbyists and students is leading to lower price points and higher capabilities for robots, radio-control vehicles, drones, and even satellites. The current state of amateur, home-built and DIY robots, quadcopters, and camera-bearing drones is providing inexpensive commercial off-the-shelf (COTS) systems to the average consumer. The price point for an individual radio-control $(\mathrm{r} / \mathrm{c})$ drone capable of taking video images at short range for later return to the sender is under $\$ 50$, but requires a human operator. Live data delivered by a user-controlled single drone is priced in the sub-\$100o range. 
The bulk of commercially available drones for amateurs are radio-control devices crossmarketed as toys and providing short-range flight control plus some video capability. These function using standard $\mathrm{r} / \mathrm{c}$ interfaces using either infrared (IR) line-of-sight control (on the sub- $\$ 100$ end), or standard FCC-licensed short range toy r/c control at $27 \mathrm{MHz}$ or $47 \mathrm{MHz}$. Sub-\$500 r/c vehicles carry no sensors or an onboard video camera that stores data to an SD card for later recovery. For autonomous simultaneous spatial sensing in our scenarios, drones are a high-risk high-cost solution that is unlikely to support substantial environmental modelling. In contrast, networked non-mobile sensors are able to cover a large spatial area over a longer time base than drones.

A combination of 3-D printing, home-built and off-the-shelf hardware, and easy sensor integration with cheap PC-on-a-chip solutions such as Arduino and Pi enables anyone with an internet connection to quickly integrate and test a unique one-off sensor. Once they have designed a sensor, they can quickly create multiple identical ones to create a deployable swarm. Our experience has shown a single college student can, in 3 months, design and improve a specific sensor design to survive a 3-gravity impact landing and provide data on a specific single variable - temperature, or seismic, or chemical information - for six hours. The hardware is cheap and easy to design and improve. The primary bottleneck is communications for a large number of deployed sensors.

Rain and water run-off measurements for irrigation have historically been measured by subjective crew assessment (Miller 2014). The RemoteTracker \$50o hand-held sensor uses acoustic Doppler, GPS, and Bluetooth transmits standardised flow measurements via wireless wide area network (WWAN) card to a central server (H2OTech 2014). Fifteen garage-built stations built by Weather Tools LLC, deployed by an entrepreneur in California, USA (Miller 2014) are spaced 7.5 mile apart, "which allows the detection of previously unseen gradients in temperature, wind, and moisture". The company hopes to "create a service that helps growers optimise crop production and resource management".

The WallyHome, another humidity, water, and temperature sensor system, uses a tiny battery and zero connectivity infrastructure to send data to an internet-connected hub. Their sensor battery lasts 10 years, and their broadcast protocol is wireless with a typical short range under 10 metres, but extends the range by resonating the existing copper power lines in the building as an antenna. The room or building data collection hub is plugged into the power lines for both power and to receive the signal, and then serves to relay that data via internet to their data collection cloud server (Greene 2014).

Within cities, hourly tracking of apartment temperatures can be used to improve municipal heating. "Heat Seek's system [in New York City] is designed to record apartment temperatures every hour and then present the data for lawyers, advocates, and inspectors." (Lipsky 2014) Their communications network is also a mesh plus hub, where each apartment has a sensor node sending data locally over a Wi-Fi network to that building's data hub, which then sends data via internet to the company central server. The sensor nodes are off-the-shelf Xbee 
modules plus temperature sensor with a price point under \$30. The central hub is a Raspberry Pi with an Xbee receiver. Including labour, they estimate $\$ 1000$ per apartment to set up monitoring (based on a Kickstarter campaign of $\$ 10,000$ to write 1,000 apartments).

A web-controlled central heating and energy monitoring system using entirely open source designs can be built for under \$US 100. Such systems send multiple sensor such as "thermistors for temperature sensing, current sensors or opto-reflective sensors for detecting motion or occupancy or even reading the dial on the gas meter. Additionally there are 4 channels of high current drivers which can be used to control relays, solenoids, dc motors etc" (Boak 2012). The power profile for remote sensors and Wi-Fi transmission to a hub is dropping to levels such that an unattended sensor can potentially last years. Ultra-low powered radios acting as internet nodes use millimetre waves to both transmit to the hub and receive power, albeit with a range of only 1-3 metres (Marshall 2014).

Additional scenarios follow this same development pathway. We do not distinguish between state agents acting in their role of disaster or environmental management, and individual organisations acting to diagnose or discover problems at a local level. The hardware is ubiquitous and available to private citizens, and requires minimal specialised knowledge. In addition, data aggregation to produce value from individual measurements is coordinated on sites such as SciStarter and Pachube, allowing individuals to contribute their local, geographically-tagged data to support larger regional or global surveys (Gertz 2012).

\section{Prototypical build}

The two models for inexpensive sensors are called, in the Arduino community, Dirt Cheap Dumb Wireless (DCDW) and Dirt Cheap Smart Wireless (DCSW). A DCDW transmits sensor data periodically and receives no communication back from the 'base station'. DCSW is an evolving field where the sensor may optionally be triggered from a base station, only reporting when queried. DCDW has lower power consumption and is the focus of our scenarios.

Commercial off-the-shelf (COTS) products fulfil the hardware design for our 'sensor pod'. Our reference model uses the MakerShed (2014) 'Wireless Sensor Node' (US \$13.99) to provide four analogue or digital sensors broadcast via an internal $433 \mathrm{MHz}$ radio transmitter with a range of 150 yards per sensor. This hardware delivers four data pod items of 8-bit sensor data at a choice of every 10 seconds, every 10 minutes, every hour, or only when a measurable event happens. It takes 20 minutes (estimated) to set up and configure with a pre-built sensor.

The detection limits for typical (US \$7) sensors include (futurelec.com) include $\mathrm{CO} 2$ readings of $0-10,000 \mathrm{ppm}$ of $\mathrm{CO} 2$ per minute, LPG readings of 200-10,000 ppm iso-butane propane, or CO sensor readings of 20-2,00oppm. Their natural gas sensor, ozone sensor, air quality sensor, methane sensor, and hydrogen sensor have performance in similar ranges.

Power needs are appropriately minimal. This sensor pod runs off a $9 \mathrm{~V}$ battery and has typical lifetimes of 15 days or longer in operations, and sleeps in between scheduled data gathers. It 
has a maximum lifetime of 76 days if read out once/hour, down to five days if read every 10 seconds (Arduino.cc 2014). Additional devices are available. Sensor data can be at resolutions from 8-bit to 14-bit, though larger sensor data packet sizes consume more power. Timing specifications, amount of user support, and survival temperature ranges for a specific system are items a designer would consider in a trade study, depending on their project's needs. Typical costs for a complete 'sensor pod' - 2 sensors, wireless transmitter, battery, case, and all supporting hardware - is US \$30 and upwards per sensor.

A sensor network requires a base station to act as a central collection 'hub' able to reroute the data via internet to a remote user. For our reference sensor pod, we configure a base station with a matching wireless receiver and Arduino microprocessor controller, which can support up to 64 of our sensor pods. Typical costs for our 'hub' are under US $\$ 100$. Power requirements remain minimal, operating from a $9 \mathrm{~V}$ battery for 2 days (Hanscr 2013). While our reference design uses an Arduino, other COTS sources offer 32 small form factor single chip controllers (Allan 2014).

A full set of 64 2-sensor pods with batteries, plus mobile base station, will cost a team approximately $\$ 2.1 \mathrm{~K}$. This provides two weeks of data taken every two minutes. The range is 150 feet, so if distributed evenly in a half-circle disk region, can cover 35,00o square feet with spacing of 500 square feet between sensors, with each sensor approximately 22 feet from each other. Coverage of a wider area requires evaluating different protocols that allow for extending the range of the sensor communications network.

\section{Sensor networking}

Within the sensor network, using off-the-shelf transmitters sharing the same frequency requires the base station be able to distinguish each individual sensor. Solutions include always-on, call-and-respond, mesh, individual channels per sensor, multiplexing, mesh networks, and individual IP addresses.

Always-on assumes all sensors are broadcasting in such a fashion that the base station receives multiple signals and has to discriminate individual sensors. Although it is trivial to design sensors that broadcast indiscriminately, in deployment the nearer sensors overwhelm the signal of further ones. Even if each sensor is theoretically distinguishable, some form of communications multiplexing is required. Our reference design can distinguish up to a maximum of 64 sensors because each sensor has a unique ID. However, data is dropped if multiple sensors communicate simultaneously.

By analogy, amateur Ham radio allows any user to transmit, but by convention tells each user not to transmit if someone else is talking. Although any Ham can be always-on, that limits receipt to the most powerful transmitter (usually the closest), and effective use of a shared frequency therefore requires some protocol for negotiating when broadcasts occur. 
Call-and-respond requires that each sensor only send a signal when queried by the base station. In this mode, a sensor is programmed to only transmit when it receives a command string from the base station. Each sensor can therefore be potentially broadcasting on the same frequency as each other sensor, since each only broadcasts when queried. This requires each sensor be equipped with a receiver as well as a transceiver, which adds to cost, power, and programming required. This mode works for $\mathrm{r} / \mathrm{c}$, ham and Wi-Fi modes. It requires minimal additional programming for the sensor modules.

Call-and-respond is particularly effective when using fixed deployment where GPS data is not needed from the sensor. If a given sensor is already tagged in the command software with a known position, the individual sensor need not include a GPS and therefore can have a lower power profile. The addition of a receiver (or transceiver) instead of just a transmitter incurs marginal overhead relative to running a GPS, and comparable power profiles are obtained in this mode.

Mesh devices not only transmit their data, but receive data from other nodes in their network and retransmit that data on further. The peer-to-peer functionality requires both sending and receiving, but allows for a larger geographical distribution of nodes from the central hub, as each node also acts as a relay.

Channel systems break up a given frequency into different coded channels, where a given sensor is sharing spectrum but expecting only one code. Toy $r / c$ devices often come equipped with up to four channels, allowing for operation of up to four identical $r / c$ devices within the same frequency. We neglect this method as infeasible for deploying the large number $(>20)$ sensors required by our scenarios, which is not addressed by off-the-shelf transceivers at our price point.

The use of Wi-Fi individual IP addresses (sometimes labelled 'the internet of things') for remote sensors is an extremely easy solution to cluster deployment for amateurs who desire a pre-built communications system that requires minimal software or networking expertise. In this model, each sensor has a unique ID and the base station gathering data. It is one specific implementation of multiplexing, and has the advantage of using an existing protocol (TCP/IP) that is easily implemented in the target hardware.

One reason for using pre-existing COTS modules is that the network topology for the multiple sensor pods will already be implemented. Given our target user is not assumed to be skilled in communications, we focus less on the specific local implementation and more on the operational limits. The topology internal to the devices must support multiple (up to 64) devices relaying to a central hub, with each individual sensor tagged with a unique identifier so we can distinguish where the data came from. In addition, we are assuming low bandwidth data being broadcast infrequently (time scales of minutes) to minimise network clashes that could lead to dropped data. This still leaves the problem of what to do with the aggregate data once it is received at the local hub. 
From a sensor pod point of view, any data item can be stamped with a sensor ID, and a data value, to which the controlling chip adds a GPS location and a pod ID. This completes the data set to provide the full capture needed by the base station. The final component is the data channel to get data from the hub to the remote data user.

\section{Hub to user communications}

Once the multiple individual sensors have relayed their data to our local hub, that hub needs to rebroadcast the full data set using a different protocol and network to our remote end user, who will be kilometres away. The easiest scenario for hub-to-user is that wired or Wi-Fi internet access is feasible from within a short distance of the sensor network. Amateur radio transmissions allow for a longer range than conventional Wi-Fi. Alternately, the hub can be fitted with GSM (capability to enable remote data delivery of the aggregated sensor data. If wired or wireless internet access exists, then our model of multiple sensors $\rightarrow$ hub $\rightarrow$ user uses ordinary internet (TCP/IP) to deliver the data to the user. Because we are investigating possible disaster areas or remote regions, however, we cannot assume an existing internet infrastructure is available.

A second technique, used in our high altitude balloons, is an amateur-band handheld radio (e.g.a \$35 Baofeng UV-5R or similar radio) to rebroadcast the signal at 4 watts VHF (136-174 $\mathrm{MHz}$ ) or 1 watt UHF (400-480MHz). This radio can be wired into the Arduino base station and requires a matching receiver station at the destination. The power lifetime for the radio is typically 10 hours, with a range of 2-5 miles or more; ranges of up to 30 miles have been achieved with a clear line of sight in outdoor tests.

Use of amateur radio frequencies requires an FCC or regional equivalent of a Technician's ham licence to operate, but if it is being used for an unmanned base station constantly transmitting, this would be already violating FCC requirements (ECFR.gov 2014). FCC Low Power Radio Station (LPRS) allow transmission of data but not for sensor use. Other wavebands have different requirements, and navigating radio licensing is often a skill set not possessed by a typical science, hobbyist, or amateur team.

One area used in amateur systems is incorporating a smartphone using G3, G4 or G5 data transmission within a standard cell phone contract. High altitude balloons (again) have used ordinary cell phones as their data transmission channel. GSM modules are available via subscription (typically US $\$ 5-10 /$ month) that attach to an Arduino or other microcontroller to provide transmission via GSM networks (Di Justo 2013). This model requires subscription to a GSM network. The cost for a single GSM or smartphone contract for a hub is feasible for amateur and citizen deployments, and is recommended for projects that do not have radio licensing knowledge or permission, and also lack internet access onsite.

Figure 1 illustrates the three most likely hub-to-user network models, arrayed in increasing cost as well as potential area coverable. A sensor network using our reference design, where each sensor communicates via short-range (150-foot) radio signals with a single hub, that then AUSTRALIAN JOURNAL OF TELECOMMUNICATIONS AND THE DIGITAL ECONOMY VOLUME 2 NUMBER 4, DECEMBER 2014 ISSN 2203-1693. Copyright (c) 2014 The Author(s). http://doi.org/10.7790/ajtde.v2n4.62 
relays to a satellite or internet provider, provides the lowest cost per-sensor but requires the sensors to be in close proximity to each other, restricting the total area covered. If the sensors have mesh capability, each sensor can be placed increasingly further from the base, extending the region covered but requiring a higher cost per sensor. The third option posits that each sensor has its own direct GSM satellite capability, removing the need for a hub and substantially increasing the potential area covered, but at greater economic cost, as it requires per-sensor GSM contracts.

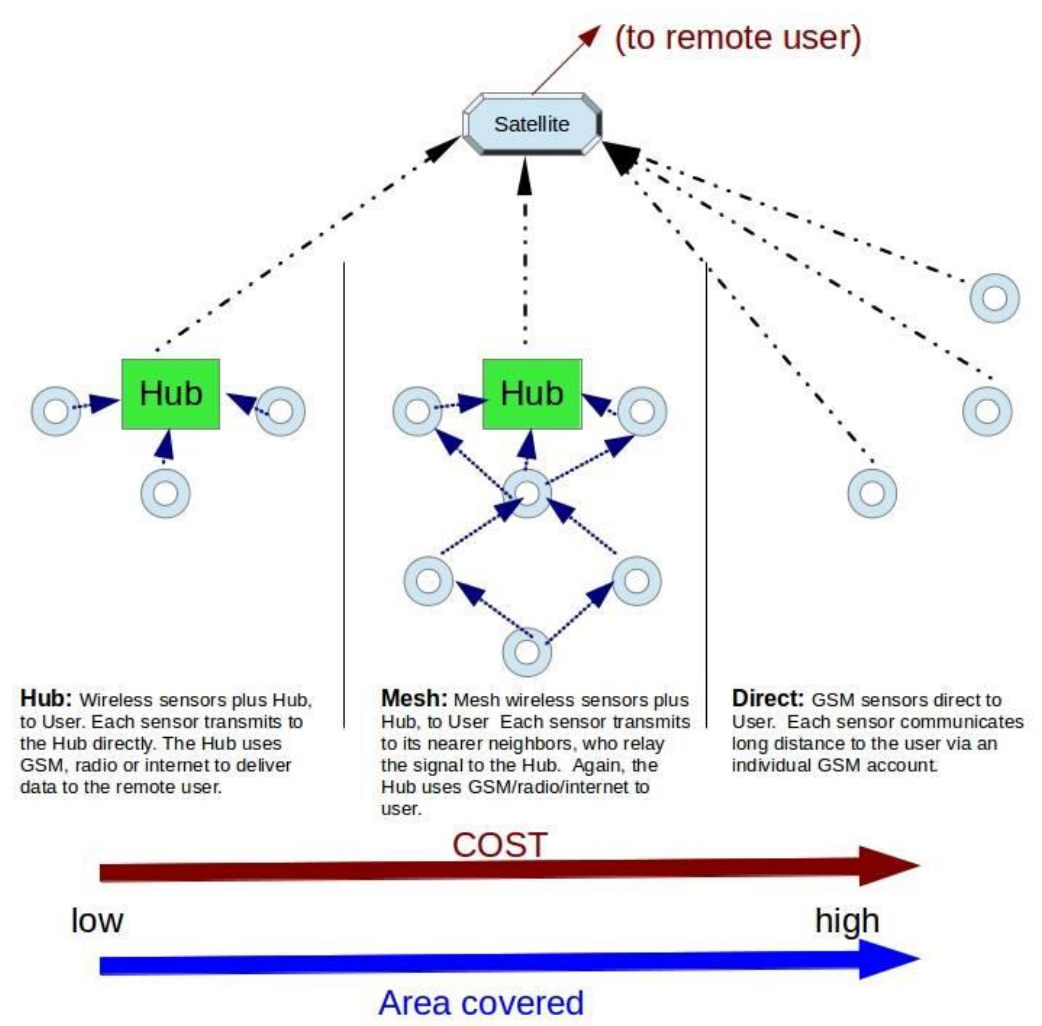

Figure 1. The three most likely hub-to-user network models

\section{Survivability and recoverability}

Placing sensors in pre-planned locations or having sensors report GPS information also ensures better recovery after the measurement period is over. The period can be defined as

a) the time needed to acquire enough data,

b) the time from start to end of crisis, or

c) the time until the sensor power has run out. 
We prefer recovery scenarios even when there is no economic driver (when the cost in staffing to recover the sensor is higher than the sensor cost), as reducing ecological waste in a good practice for scientists and monitors to encourage. One sensor in a forest might seem minor; a network of 64 is an annoyance; three universities all deploying 2000+ sensors is an ecological mistake.

Use of an onboard GPS for a sensor pod eliminates the need for specific placement and allows for more rapid deployment, potentially by non-technicians with no finesse needed. A GPS typically adds $\$ 30$ to the cost and provides exact positions (within the US, accuracy to 3.5 metres). A robust throwable or droppable sensor pod that provides its own position information is recommended because it provides flexibility in multiple scenarios where access to the terrain or locale may be more difficult than expected. Difficulties can include regions that are flooded, have traffic blocked away, are restricted access, or are geologically precarious for humans to access.

Deployment of sensors also requires cases sufficient to survive the environment. For benign environments exposed only to ordinary weather, a plastic case suffices. Sensors designed to operate in hostile environments, including in lava, in fire, and under water, are beyond the scope of this article. We do note that a good amount of data for situations such as the growth of a forest fire can be provided with a destructible sensor that simply stops broadcasting when the fire reaches it, thus providing the necessary data point on fire line expansion.

Creating a shell for the cheap sensors can be done in small batches using existing 3-D printing technology. The casing of our device is created using a 3-D printer in order to make a form suitable for the environment expected. Pods dropped or launched will have to survive an 'eggdrop' type test to ensure the electronic contents survive. Any pod expecting water or chemical contaminate will have to be tested to survive that environment. Pods to measure fire can be presumed to only survive until the outer casing melts, at which point ceasing of transmission due to casing damage is itself the final data point. Our best guidelines are that 3 -D printing enables rapid iteration of testing until a given casing is shown to survive (Antunes et al 2013).

For our lab tests our requirement was ' 3 -D cube at $10 \mathrm{~cm} \times 10 \mathrm{~cm} \times 10 \mathrm{~cm}$ able to withstand the static weight of a person, a dynamic drop from 1 metre, temperatures down to -6oC, and pressures down to a soft vacuum', as our cubes were designed to support high altitude balloon tests. It took five iterations by a single student to create a suitable 3-D shell. We therefore suggest shell design is a straightforward engineering problem easily solved through appropriate iteration and testing.

\section{Implementation challenges}

Social and legal concerns are a possible implementation challenge. Di Justo and Gertz (2013) note that one should "not deploy your gadget in public without official permission", citing two cases where well-intentioned citizens were arrested for placing devices that police later 
mistook as potential bomb threats. An unexpected box of electronics with a blinking light in public is, in their assessment, a risk. They suggest three strategies to mitigate causing alarm.

Firstly, official permission can sometimes be obtained by regional Parks Departments or by the government or private entity that owns the land. If the project is educational signed support by your institution must be obtained. Secondly, when deploying sensors in a community, discuss the project with the community before implementing the research.

Finally, in addition to communicating to the public and police prior to deploying multiple electronic devices, we also recommend clear labelling of your devices. However, also note that labelling alone may not suffice, as demonstrated by the example of students at CarnegieMellon University, who had to answer to police and had their equipment confiscated despite clear labelling (Di Justo 2013). Despite having solved the technical challenges, potential legal and human risks remain.

\section{Conclusions}

Networked deployable low-fidelity environmental sensors are an active field of study as well as entrepreneurship. We assert that an individual or small group can accurately sample a moderate region, on the scale of a kilometre or so, with sensors that report every few minutes over a several day period to a base station located kilometres away. The cost per sensor is well under US $\$ 100$ and requires no special engineering talent. Covering small geographic region using simple sensors that relay data to a local hub, which then communicates that data to a remote user using GSM, is economically and technologically simple and available to general citizens. This enables citizen and activist groups as well as research entities to tackle smallscale projects for specific targeted goals.

The technology is available for amateurs and non-engineers through use of DIY resources, inexpensive pre-built hardware, 3-D printing, and conventional internet. Design of a custom project in largely an exercise in component selection, followed by integration and testing. The sample build presented is documented and freely available via internet resources. The primary limitation in deploying a large number of sensors is ensuring there is a viable communications channel from near the remote site, to the home base of the user.

For situations ranging from fires and crises through to urban monitoring, cheap deployable sensors fill a gap between existing satellite-based and large autonomous sensor deployment by government and commercial entities, and single-user sampling via individuals or drones. The ease of design and deployment should be approached with an eye towards minimising adding technological pollution to an environment, and also needs to follow existing communications licensing and use policies. Within this framework, we feel that the growth of this technology is a positive force carrying minimal risk in enabling new groups to monitor measurable situations and potentially improve local environmental conditions. 


\section{References}

Allan A. 2014. 'Which Board is Right for Me'. Make Magazine. 36. Available from: http://makezine.com/magazine/make-36-boards/which-board-is-right-for-me/

Antunes, A; Walters, A.; Bormanis, M.; Schrenk, R. 2014. 'Constrained Multi-Altitude Design Using 3D Printed CubeSat Shells'. AIAA SciTech 2014. Available from: https://www.aiaa.org/Secondary.aspx?id=20342.

Arduino.cc. 2014. http://forum.arduino.cc/index.php?topic=63138.0;wap2

Boak, K. 2012. 'Building Wireless Sensing Networks- using Open Source Hardware'. Nanode LLC. Available from: http://www.nanode.eu/news/building-wireless-sensing-networks-using-opensource-hardware/

Di Justo, P. and Gertz, E. 2013. 'Atmospheric Monitoring with Arduino'. O'Reilly/Maker Press.

ECFR. 2014. US Government Printing Office. Available from: http://www.ecfr.gov/cgi-bin/textidx?SID=e593edgf147a6ee87a8803do5b9667c9\&tpl=/ecfrbrowse/Title47/47cfr95 main 02.tpl

Gertz, E. and Di Justo, P. 2012. 'Environmental Monitoring with Arduino'. O'Reilly/Maker Press. GPS.gov. 2014. http://www.gps.gov/systems/gps/performance/accuracy/

Greene, K. 2014. 'Nest Competitor Monitors Your House's Leaks on the Cheap'. MIT Technology Review. Available from: http://www.technologyreview.com/news/523741/nest-competitor-monitorsyour-houses-leaks-on-the-cheap/

H2OTech.2014. Available from: http://www.h2otechonline.com/innovations/remotetracker/faq/

Hanscr. HWStartup. 2013. 'How to run an Arduino on a 9V battery for Weeks or Months'. Available from: http://hwstartup.wordpress.com/2013/03/11/how-to-run-an-arduino-on-a-gv-battery-forweeks-or-months/

Lipsky, J. 2014. 'Sensors Build Case Against Cold'. EE Times. Available from: http://www.eetimes.com/document.asp?doc id $=1324130$

Marshall, P. 2014. 'Ant-sized radios for the Internet of Things'. Government Computing News (GCN). Available from: http://gcn.com/blogs/emerging-tech/2014/og/iot-radio-chip.aspx

Middleton, C. 2014. 'Building a Digital Society'. Australian Journal of Telecommunications and the Digital Economy, Vol 2, No 1, Article 27. Available from:

http://doi.org/10.7790/ajtde.v2n1.27

Miller, E. 2014. 'North State entrepreneurs innovate for efficient water use.' North Valley Correspondent. Available from: http://www.centralvalleybusinesstimes.com/stories/o01/?ID=26826

MakerShed. 2014. See http://www.makershed.com/products/wireless-sensor-node 